\title{
Extreme ultraviolet and soft $X$-ray imaging using compact laser- plasma sources based on a double stream gas-puff target
}

\author{
Przemysław W. Wachulak ${ }^{1, *}$, Andrzej Bartnik ${ }^{1}$, Jerzy Kostecki ${ }^{1}$, Lukasz Wegrzynski $^{1}$, Tomasz Fok ${ }^{1}$, \\ Roman Jarocki ${ }^{1}$, Mirosław Szczurek ${ }^{1}$, and Henryk Fiedorowicz ${ }^{1}$ \\ ${ }^{I}$ Institute of Optoelectronics, Military University of Technology, Kaliskiego 2, 00-908 Warsaw, Poland,
}

Received July 16, 2013; accepted December 17, 2013; published December 31, 2013

\begin{abstract}
New developments in science and technology require imaging tools and techniques capable of imaging with increased contrast and high spatial resolution, such as extreme ultraviolet (EUV) and soft X-ray (SXR) microscopy. In this paper, we report on our recent activities related to various imaging techniques in the EUV and SXR region of electromagnetic spectrum, employing a double stream gas-puff target source. Those techniques have been successfully used for studies of morphology of thin silicon membranes coated with $\mathrm{NaCl}$ crystals, samples composed of $\mathrm{ZnO}$ nanofibers and biological samples.
\end{abstract}

Decreasing the illumination wavelength is a direct way to improve spatial resolution in photon-based imaging systems, since the spatial resolution $\delta$ is directly proportional to the illumination wavelength $\lambda[1]$, as can be seen in Eq. (1):

$$
\delta=\frac{0.61 \cdot \lambda}{N A},
$$

where $N A$ is the numerical aperture of the optics used to form images. Thus the worldwide interest in microscopy at extreme ultraviolet (EUV) and even shorter, soft X-ray (SXR) wavelengths, photon-based EUV/SXR microscopy is currently capable of reaching resolutions down to $12 \mathrm{~nm}$ using synchrotron radiation [2]. However, the complicacy and extreme financial demands associated with measurements using synchrotron radiation lead to the existing demand for more affordable sources of shortwavelength radiation. The introduction of compact sources of bright EUV and SXR light paved the way for the development of tabletop microscopes that can render images of nanoscale objects with exposures as short as a few seconds and spatial resolution approaching that of synchrotron-based microscopes [3-6].

The goal of this paper is to present our recent activities in extreme ultraviolet and soft X-ray imaging at the Institute of Optoelectronics, Military University of Technology, Warsaw, Poland. Two microscopes will be presented: an EUV microscope, capable of imaging nanostructures with $\sim 50 \mathrm{~nm}$ half-pitch spatial resolution and an SXR microscope operating in the so called "water-

*e-mail: wachulak@gmail.com window" spectral range with a spatial resolution down to 850nm.

The EUV microscope was equipped with an ellipsoidal mirror with an $\mathrm{Mo} / \mathrm{Si}$ coating to focus EUV radiation onto an object. A Fresnel zone plate (FZP) objective was used to form a magnified image onto a EUV-sensitive CCD camera in the transmission mode. The use of the gas puff target eliminates the debris production problem associated with solid targets. Quasi-monochromatic EUV radiation, which is required for the use of Fresnel optics, was produced by spectral selection of a single line emitting at $13.8 \mathrm{~nm}$ wavelength from broad band argon plasma radiation. Different test objects (samples) were imaged with this setup: $\mathrm{Cu}$ mesh with a thickness of $\sim 4 \mu \mathrm{m}$ [7] and carbon foil, 70nm thick, with holes [8], where the EUV images have been obtained with the half-pitch spatial resolution approaching $\sim 50 \mathrm{~nm}(3.7 \lambda)$ in a very compact setup. More details about the setup can be found in [7, 8]. The laser plasma EUV source used in the experiment was optimized for quasi-monochromatic emission in the 13$14 \mathrm{~nm}$ wavelength range, described in detail elsewhere [9]. This source has an advantage over other compact sources due to the fact that simply by choosing a suitable gaseous target it is possible to change both the peak emission wavelength and the inverse relative bandwidth of the emission from the plasma. Moreover, the gaseous target does not produce any debris associated with solid targets. Ar plasma was produced by $\mathrm{Nd}$ :YAG laser (Eksma) irradiation of a gaseous target with a pulse duration of $4 \mathrm{~ns}$, wavelength $1064 \mathrm{~nm}$, and energy $0.74 \mathrm{~J}$. The plasma radiates in a very broad range of wavelengths, dominantly in the EUV range $(5-50 \mathrm{~nm})$. EUV radiation from the plasma was both focused and spectrally filtered by a $\mathrm{Mo} / \mathrm{Si}$ multilayer ellipsoidal mirror (condenser) developed in collaboration with Reflex s.r.o. (mirror substrate) and Fraunhofer Institut für Angewandte Optik und Feinmechanik (coating). The multilayer coating was optimized for the $13.5+/-0.5 \mathrm{~nm}$ (FWHM) wavelength range and 45 degrees incidence angle. The in-band $(\lambda=13$ $14 \mathrm{~nm})$ photon flux was $(8.8 \pm 0.5) \cdot 10^{10}$ photons per pulse in a horizontally elongated spot with a size of 
$1.09 \times 0.39 \mathrm{~mm}^{2} \quad(\mathrm{FWHM})$ [8]. That corresponds to the energy of $1.29 \mu \mathrm{J} /$ pulse. To eliminate longer wavelengths $(\lambda>18 \mathrm{~nm})$, a 100 -nm-thick, $\mathrm{Zr}$ filter (Lebow) was used. The resulting quasi-monochromatic spectrum has two main peaks at $13.793 \mathrm{~nm}$ and $13.844 \mathrm{~nm}$ wavelength transitions in $\mathrm{Ar}^{\mathrm{VIII}}$ [10]. The inverse relative bandwidth (FWHM) of the filtered spectrum is $\lambda / \Delta \lambda=140$ at $\lambda=13.84 \mathrm{~nm}$. Different objects: meshes, thin films and nanofibers, were imaged with this system using a FZP objective (Zone Plates Ltd.) onto a EUV sensitive CCD camera iKon-M (Andor) with $1024 \times 1024$ pixels and $13 \times 13 \mu \mathrm{m}^{2}$ pixel size. The diameter of the FZP is $\mathrm{D}=200 \mu \mathrm{m}$, number of zones $\mathrm{N}_{\mathrm{FZP}}=1000$ and an outer zone width $\Delta \mathrm{r}=50 \mathrm{~nm}[11]$. In the case of "binary" transmission test objects such as $\mathrm{Cu}$ mesh, 25-50 EUV pulses at $2 \mathrm{~Hz}$ repetition rate were necessary to obtain a single image [7]. The CCD camera was cooled down to $-20^{\circ} \mathrm{C}$ to decrease intrinsic noise during the image acquisition.

Some examples of images obtained with the EUV microscope are presented in Fig. 1. Polycrystalline layers of $\mathrm{NaCl}$ deposited from water solution on top of a $15 \mathrm{~nm}$ thick silicon membrane, imaged with optical microscope at $100 \times, \mathrm{NA} \sim 1$ (a) and EUV microscope (b) are compared. More details about this work are presented in [12].
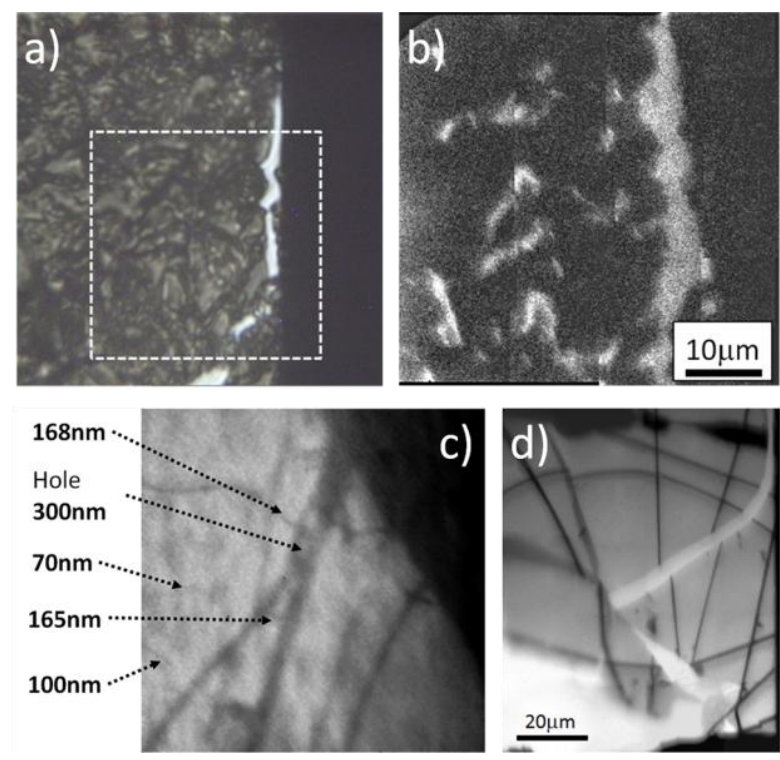

Fig. 1. Example of images of various objects obtained with the use of EUV microscope: cracks in $\mathrm{NaCl}$ layer (b) compared to a visible light microscopy image (a), $\mathrm{ZnO}$ nanofibres deposited on top of a gold mesh (c) and similar nanofibres on top of cracked silicon nitride membrane (d).

Moreover, $\mathrm{ZnO}$ nanofibers were deposited on top of a gold mesh and thin silicon nitride membrane and later imaged using the EUV microscope. A small, $\sim 20 \times 20 \mu \mathrm{m}^{2}$ in size, fragment of the sample, imaged at $13.8 \mathrm{~nm}$ wavelength with the EUV microscope, 100 EUV pulses exposition, 690× magnification, NA 0.138, can be seen in Fig. 1c) and was reported in [13]. Similar nanofibres deposited on top of a cracked silicon nitride membrane are presented in Fig. 1d). Cracks and nanofibers as small as $100 \mathrm{~nm}$ are visible.

Photon-based imaging at short wavelength vs. electron, or recently neutron, imaging has additional advantages due to different interaction of photons with matter. Atomic resonance frequencies, leading to very high absorption coefficients at EUV and SXR wavelengths, provide an enhanced optical contrast. A particularly suitable range of wavelengths for biological imaging is the so-called "water-window" spectral range. X-ray sources, emitting in the "water-window" region between 2.3 and $4.4 \mathrm{~nm}$ wavelength [14], are thus important for biological applications. High contrast in this spectral range is obtained due to a difference in the absorption of different constituents of biological specimens. While water, present in the sample, has a relatively small absorption coefficient in this spectral range, carbon, due to much higher absorption, gives a very good contrast in the image. Thus this spectral range is perfectly suitable for imaging biological samples.

We successfully combined the advantages of employing a compact, laboratory type laser-plasma short wavelength source, based on a gas puff target, emitting incoherent radiation, with the "water-window" spectral range from argon and nitrogen plasmas and a Wolter typeI reflective objective. This unique combination is suitable for biological imaging, and allows to develop small size microscopy setups, which might be used in various fields of science and technology. More details about both microscopes can be found elsewhere [15-16]. The "waterwindow" microscope was equipped with a previously mentioned laser plasma gas puff target source, optimized for efficient emission in the "water-window" range. The plasma radiates in a very broad range of wavelengths, including the SXR region and by using additional spectral filtering it is possible to tailor the spectral emission of the source. EUV radiation from the plasma was collected and focused by an ellipsoidal, axi-symmetrical nickel coated condenser mirror, developed by Rigaku, Inc. The condenser is a broad-band optic, capable of efficiently reflecting radiation from the EUV range down to SXR region. To spectrally narrow the emission from argon or nitrogen plasma a $200 \mathrm{~nm}$ thick, free-standing titanium filter (Lebow) was used. The sample is imaged onto a SXR sensitive back-illuminated, $1024 \times 1024$ pixels, $13 \times 13 \mu \mathrm{m}^{2}$ pixel size, CCD camera (i-Kon, DO-934N model, from Andor) by a Wolter type-I reflective objective [17], composed of two axially-symmetric ellipsoidal and hyperboloidal nickel coated mirrors. In our current setup the magnification of the objective is equal to $14.6 \times$. Depending on gas, the spectrum illuminating the 
sample is broad (from $\lambda=2.8-5 \mathrm{~nm}$ for argon plasma), or monochromatic $(\lambda=2.88 \mathrm{~nm}$ from nitrogen plasma). Typical images of the metal meshes, obtained under argon plasma illumination, filtered by a $200 \mathrm{~nm}$ thick Ti filter, are shown in Fig. 2a), for a square mesh and b) for a rectangular mesh, respectively. An exemplary image of a biological object (onion skin cells) is presented in Fig. 2c).
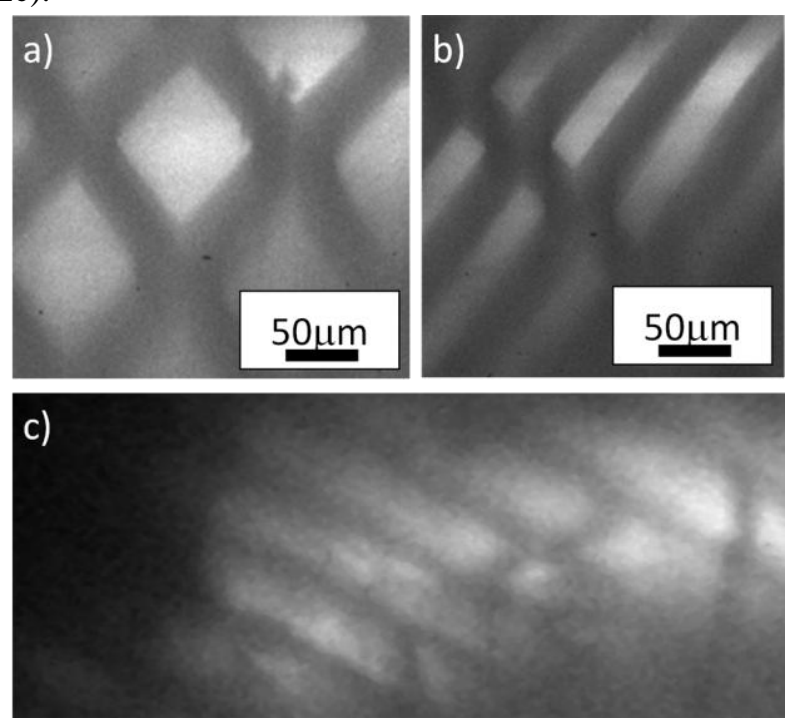

Fig. 2. SXR images of the test objects - metal meshes (a-b), exposure 10 SXR pulses (1 second exposure time), image of onion skin cells (c), exposure 100 SXR pulses (10 seconds), obtained in the "water-

window" spectral region. Biological sample is up to $40 \mu \mathrm{m}$ thick.

The required exposure for high contrast test objects (meshes) was of the order of $10 \mathrm{SXR}$ pulses, so for the $10 \mathrm{~Hz}$ repetition rate of our source, the exposure time was equal to 1 second. In the case of a much thicker biological sample, the exposure of 200 SXR pulses was required. High water transmission in the ,water-window” spectral range opens up the possibility to perform imaging of samples $\sim 40 \mu \mathrm{m}$ thick.

Knife edge resolution measurements indicate statistical half-pitch spatial resolution equal to $1.1 \mu \mathrm{m}$ for argon plasma illumination [15] and 850nm for nitrogen plasma source [16].

In conclusions, we have demonstrated the imaging of nanostructures using a desk-top EUV transmission microscope based on laser-produced plasma from an argon-based gas-puff target and Fresnel optics. The short attenuation length of EUV photons in almost any material can be utilized to perform imaging with an enhanced optical contrast and yet, due to the intermediate photon energy, cause less radiation damage than hard X-ray beams. Thus, EUV microscopy can be employed in thin layer studies providing information complementary to optical and SEM microscopy. We also presented the results of $\mathrm{ZnO}$ nanofibers imaging, showing improved spatial resolution and good optical contrast of this material at EUV wavelengths. Another microscopy system that was presented - "water-window" compact, desk-top microscope, employed a laser-plasma SXR source based on an argon or nitrogen double stream gas puff target and a Wolter type-I objective. This system allows capturing magnified images of objects, with a magnification of $\sim 15 \times, 850 \mathrm{~nm}$ half-pitch spatial resolution and exposure time as low as a few seconds.

The research was supported by the Foundation for Polish Science, HOMING 2009 Programme, Grant number HOM/2009/14B and by the National Science Centre, award number DEC-2011/03/D/ST2/00296. We thank Ladislav Pina and Thorsten Feigl for their help in the preparation of EUV optics. We would also like to thank René Hudec from the Astronomical Institute, Academy of Sciences of the Czech Republic for the Wolter type-I SXR objective.

\section{References}

[1] D. Attwood, Soft X-Rays and Extreme Ultraviolet Radiation, (Cambridge University, Cambridge, England, 1999).

[2] W. Chao, J. Kim, S. Rekawa, P. Fischer, E.H. Anderson, Opt. Expr. 17, 20, 17669 (2009),

www.opticsinfobase.org/oe/abstract.cfm?uri=oe-17-20-17669

[3] G. Vaschenko, et al., Opt.Lett. 31, 1214-1216 (2006), http://www.opticsinfobase.org/ol/abstract.cfm?uri=ol-31-9-1214

[4] C.A. Brewer, et al., Opt. Lett. 33(5), 518, (2008), http://www.opticsinfobase.org/ol/abstract.cfm?uri=ol-33-5-518

[5] K.W. Kim, et al., Phys. Med. Biol. 51, N99-N107 (2006).

[6] L. Juschkin, R. Freiberger, K. Bergman, J. Phys.: Conf. Ser. 186, 012030 (2009).

[7] P.W. Wachulak, A. Bartnik, H. Fiedorowicz, Opt. Lett. 35(14), 2337 (2010),

http://www.opticsinfobase.org/ol/abstract.cfm?uri=ol-35-14-2337

[8] P.W. Wachulak, A. Bartnik, H. Fiedorowicz, J. Kostecki, Opt. Exp. 19(10), 9541 (2011), www.opticsinfobase.org/oe/abstract.cfm?uri=oe-19-10-9541

[9] P.W. Wachulak, et al., Appl. Phys. B 100(3), 461 (2010), www.opticsinfobase.org/oe/abstract.cfm?uri=oe-19-10-9541

[10] R.L. Kelly, J. Phys. Chem. Ref. Data, 16, suppl. 1 (1987).

[11] The ZP was fabricated by Zone Plates Ltd., using electron beam lithography in 220nm thick PMMA (polymethyl methacrylate) layer spin-coated on top of a $50 \mathrm{~nm}$ thick silicon nitride $\mathrm{Si}_{3} \mathrm{~N}_{4}$ membrane.

[12] P.W. Wachulak, A. Bartnik, H. Fiedorowicz, D. Panek, P. Bruza, Appl. Phys. B 109, 105 (2012), http://link.springer.com/article/10.1007\%2Fs00340-012-5125-3

[13] P.W. Wachulak, et al., Rad. Phys. Chem. (2013) - in press, http://dx.doi.org/10.1016/j.radphyschem.2013.02.019

[14] L.B. Da Silva, et al., Science 258, 269 (1992).

[15] P.W. Wachulak, et al., Appl. Phys. B 111(2), 239 (2013), http://link.springer.com/article/10.1007\%2Fs00340-012-5324-y

[16] P.W. Wachulak, et al., Nucl. Instr. Meth. B (2013) - in press, DOI: $10.1016 /$ j.nimb.2013.06.001.

[17] H. Wolter, Ann. Physik 10, 286 (1952). 